\title{
Quantum coherent dynamics of molecules: A simple scenario for ultrafast photoisomerization
}

\author{
Daniel P. Aalberts, ${ }^{1, *}$ M. S. L. du Croo de Jongh, ${ }^{2}$ Brian F. Gerke, ${ }^{1, \dagger}$ and Wim van Saarloos ${ }^{2}$ \\ ${ }^{1}$ Physics Department, Williams College, Williamstown, Massachusetts 01267 \\ ${ }^{2}$ Instituut-Lorentz, Universiteit Leiden, P.O. Box 9506, 2300 RA Leiden, The Netherlands
}

(Received 11 November 1999; published 28 February 2000)

\begin{abstract}
We analyze the coherent dynamics of optically excited alkenes in a fully correlated $3 d$ tight-binding model with extended Hubbard interactions. The scenario that emerges is that the steric repulsive interactions are the driving force behind ultrafast cis-trans photoisomerizations. This resolves the apparent discrepancy between values for the torsional stiffness obtained from band-structure potentials and from vibrational spectra. The mechanism is illustrated in quantitative detail for ethylene and is also shown to yield a promising scenario for the coherent dynamics of molecules like retinal.
\end{abstract}

PACS number(s): 82.50.-m, 87.15.He, 82.40.Js, 34.20.Cf

Cis-to-trans isomerizations (see Fig. 1) are important photochemical reactions for polyenes. These reactions were already demonstrated 40 years ago [1] to be the primary reaction in vertebrate vision; nevertheless, a general and intuitive understanding of the mechanism has remained elusive. Recent experiments have shown that molecular shape-changing reactions are extremely fast and efficient; for example, $200 \mathrm{fs}$ [2] and a quantum efficiency of $65 \%$ [3] for photoisomerization of retinal in the visual pigment rhodopsin, and $30 \pm 15 \mathrm{fs}$ for the internal conversion of ethylene [4]. Femtosecond experiments indicate coherent and coupled dynamics of electronic and spatial degrees of freedom [5].

The theory of ultrafast processes has not yet caught up with the extraordinary experimental developments. Model elements are not in dispute and the ground-state properties have been calculated in approximations described below; however, these methods are unable to treat fully correlated optically excited wave functions, and that makes dynamical calculations suspect. Moreover, they have so far not been able to yield a convincing intuitive picture of the photoisomerization mechanism. But now, by combining familiar interactions with the correct quantum-correlated states, we find a simple mechanistic picture for ultrafast photoisomerization. While the accepted view is that bond order changes before isomerization [6,7], our additional insight is that photoexcitation so weakens double bonds' strength that the molecule succumbs to twisting forces arising from steric repulsions. This remarkably simple, robust, and elegant mechanism for photoisomerization provides a promising starting point for studying other types of coherent dynamics, including the suggestion $[8,9]$ that the ultrafast isomerization of retinal is associated with soliton pair formation.

The theory of molecules in their electronic ground states relies on (1) optimizing the electron density function [10], (2) using single-electron or mean-field approximations [Hartree-Fock, molecular orbital, self-consistent-field (SCF),

\footnotetext{
*Corresponding author.

${ }^{\dagger}$ Present address: Emmanuel College, Cambridge University, Cambridge CB2 3AP, United Kingdom.
}

or configuration-interaction $\mathrm{SCF}$ ] to calculate energy bands [6,11-15], or (3) inferring phenomological force fields from structural data or vibrational spectra in a molecular mechanics approach [16].

To calculate the dynamics of optically excited states is a hugely more difficult problem. Difficulties in treating ultrafast dynamics perhaps have arisen because (i) products of single-electron SCF states produce wave functions neglecting correlations, (ii) highest occupied molecular orbital (HOMO) to lowest unoccupied molecular orbital (LUMO) excitations also neglect correlations, (iii) only $\pi$ electronic effects were included in the effective energy used to calculate dynamics [12], or (iv) the $(3 N-5)$ degrees of freedom were condensed to one predefined reaction coordinate, thereby excluding the effects of the strong coupling between electronic and spatial degrees of freedom that has been shown to be so important in polyacetylene.

In this paper, we (i) calculate fully correlated electronic ground states, (ii) use a second quantized operator formalism to create optically excited states, (iii) include the essential contributions of $\sigma$-bonded and nonbonded interactions in the dynamics, and (iv) keep all carbon dynamical degrees of freedom. We shall leave discussion of deexcitations and surface crossings $[6,11]$ for the present to concentrate on how photons can instigate spatial dynamics.
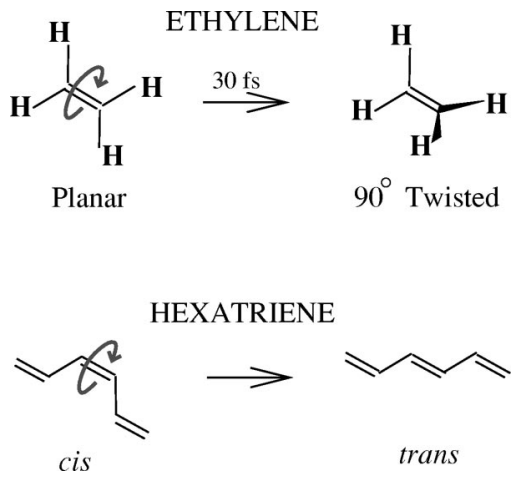

FIG. 1. Photoisomerizations are reactions in which molecules change their conformation after the absorption of a photon. (Hydrogens are not depicted for hexatriene.) 
For ease of presentation, after briefly describing the molecular model we shall first discuss the photoexcitation of ethylene, as the basic mechanism can be elucidated analytically in that case. We then turn to longer polyenes.

The model is fashioned from basic and unquestionable components of the Pariser-Parr-Pople (PPP) variety [6,11$15]$. Because the fully occupied $\sigma$ bonds are spectroscopically inert while the higher-energy half-filled $\pi$ bonds are highly responsive to external electric fields (such as the photon's) and highly coupled to the spatial coordinates of the nuclei, the model is built on a proper separation into classical and quantum constituents.

The extended Hubbard (EH) model is used to treat the $\pi$ electrons. This model includes both the physics incorporated in the SSH model for polyacetylene [14] and correlation effects, in view of the fact that mean-field theories and noninteracting models are notoriously unreliable in one dimension. With recent conceptual and computational advances, it is now feasible technically to analyze quantum correlated systems using exact diagonalization or the density matrix renormalization group [17]. Fully correlated methods such as these allow one to preserve the coherence of the states. Like others [13-15], we use molecular mechanics (MM) parabolic (springlike) potentials for $\sigma$ bonds. We will write the model in second quantization notation and, to emphasize our method, refer to this realistic and fairly quantitative model as extended Hubbard molecular mechanics (EHMM).

The EHMM correlated $\pi$ electron Hamiltonian is

$$
\begin{aligned}
H_{\pi}= & \sum_{j, s} T_{j}\left[c_{j, s}^{\dagger} c_{j+1, s}+c_{j+1, s}^{\dagger} c_{j, s}\right]+\sum_{j} U\left(n_{j \uparrow}-\frac{1}{2}\right)\left(n_{j \downarrow}-\frac{1}{2}\right) \\
& +\sum_{j>k} V_{j, k}\left(n_{j}-1\right)\left(n_{k}-1\right),
\end{aligned}
$$

with

$$
\begin{gathered}
T_{j}=-\left(t-\alpha u_{j}\right) \cos \theta_{j}, \\
u_{j}=\left|\mathbf{r}_{j}-\mathbf{r}_{j+1}\right|-a_{0}, \\
V_{j, k}=U\left(1+\eta\left|\mathbf{r}_{j}-\mathbf{r}_{k}\right|^{2}\right)^{-1 / 2} .
\end{gathered}
$$

The nuclear coordinate of atom $j$ is $\mathbf{r}_{j}$. The first term in Eq. (1) accounts for the hopping of electrons between atoms $j$ and $(j+1)$ at a rate which can be reduced by pulling the atoms apart from their average distance $a_{0}=1.4 \AA$ or by twisting the $\pi$ orbitals away from the parallel configuration, where $\theta=0^{\circ}$. The Coulomb penalty for electron-electron interactions is incorporated via the Hubbard $U$ term and the extended-Hubbard $V$ term. With $\eta=(U / 14.397 \mathrm{eV} \AA)^{2}$, this is the Ohno parametrization [18] for the extended Hubbard model; but other parametrizations may also be used successfully.

To treat photoexcitation, we use the photon interaction operator $H_{\mathrm{int}}=\mathbf{A} \cdot \mathbf{J} / c$ [19] that couples the electric field of the photon to the $\pi$ electrons. The vector potential points in the direction of the electric field and is proportional to photon creation and annihilation operators, $\mathbf{A}=\mathbf{E}\left[\cdots a^{\dagger}\right.$ $+\cdots a]$. The current operator $\mathbf{J}$,

$$
\mathbf{J}=\frac{i}{\hbar} \sum_{j, s} T_{j}\left(\mathbf{r}_{j}-\mathbf{r}_{j+1}\right)\left[c_{j, s}^{\dagger} c_{j+1, s}-c_{j+1, s}^{\dagger} c_{j, s}\right],
$$

determines the response of the $\pi$ electrons. The electronic state following photoexcitation is $|\mathrm{OpEx}\rangle \propto \mathbf{J}|\mathrm{GS}\rangle$.

The nuclear Hamiltonian due to $\sigma$ bonding is [15]

$$
\begin{aligned}
H_{\sigma}= & \frac{1}{2} K_{\sigma} \sum_{j} u_{j}^{2}+\frac{1}{2} K_{120} \sum_{j} \phi_{j}^{2}+\frac{1}{2} \sum_{j} M_{j}\left(\frac{d \mathbf{r}_{j}}{d t}\right)^{2} \\
& +\sum_{k, \ell} D e^{-A R_{k \ell}},
\end{aligned}
$$

where $\phi$ is the angle a carbon's $\sigma$ orbitals deviate from the ideal $120^{\circ}$. We include the translational kinetic energy of the ions (C's and H's) but neglect that of the electrons because of their light mass. As in [6,11-15], we assume that orbitals follow the ionic positions instantaneously.

The final term in Eq. (3) represents steric interactions between filled orbitals; it plays a decisive role in the mechanism we wish to elucidate. We take the steric energy to be an exponential function of the distance $R_{k}$ between centers of nonbonded $\sigma$ orbitals. The exponential form is not essential but reflects the undisputed fact that, at close range, filled orbitals repel.

Let us now illustrate our photoisomerization scenario with the ethylene molecule $\left(\mathrm{C}_{2} \mathrm{H}_{4}\right)$. In this context, a complete analysis is possible and the role of the sterics is quite easily seen.

The spin-zero basis vectors for a two-site system are

$$
\begin{array}{cc}
|20\rangle=c_{1 \uparrow}^{\dagger} c_{1 \downarrow}^{\dagger}|\rangle, \quad|\uparrow \downarrow\rangle=c_{1 \uparrow}^{\dagger} c_{2 \downarrow}^{\dagger}|\rangle, \quad|\downarrow \uparrow\rangle=c_{2 \uparrow}^{\dagger} c_{1 \downarrow}^{\dagger}|\rangle, \\
|02\rangle=c_{2 \uparrow}^{\dagger} c_{2 \downarrow}^{\dagger}|\rangle,
\end{array}
$$

Normal ordering has down operators acting first. With this basis sequence,

$$
H_{\pi}=\left(\begin{array}{cccc}
U & T & T & 0 \\
T & V & 0 & T \\
T & 0 & V & T \\
0 & T & T & U
\end{array}\right) .
$$

The eigenvectors are

$$
\begin{gathered}
\left.|\mathrm{GS}\rangle=(f, g, g, f)^{T}, \quad \mid \text { triplet }\right\rangle=\frac{1}{\sqrt{2}}(0,1,-1,0)^{T}, \\
|\mathrm{OpEx}\rangle=\frac{1}{\sqrt{2}}(1,0,0,-1)^{T}, \quad|\mathrm{AB}\rangle=(g,-f,-f, g)^{T},
\end{gathered}
$$

with $|f| \ll|g|$. The dependence of the eigenvalues on torsional angle is shown in Fig. 2(a). 


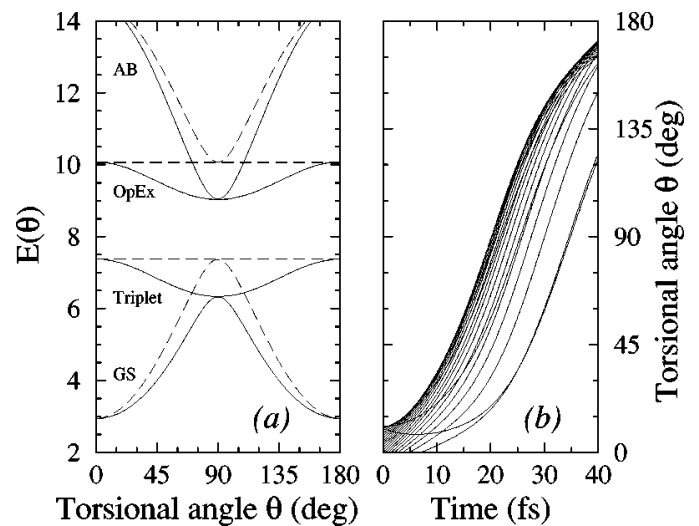

FIG. 2. (a) The energy eigenstates of ethylene are ground, triplet, optically excited, and antibonding. The contribution from the $\pi$ electrons alone is depicted with dashed lines. Steric effects favor twisting to $\theta=90^{\circ}$, giving the total energy as depicted with solid lines. In the ground state, the torsional stiffness is reduced by the steric effects, while in the optically excited state, the steric interactions become dominant and induce the molecule to twist. (b) At time $t=0$ an ethylene molecule is photoexcited. An ensemble consistent with zero-point motion in the ground state is used for the initial conditions. The time to twist to $\theta=90^{\circ}$ compares well to the measured internal conversion time scale $30 \pm 15$ fs [4].

In ethylene, $H_{\text {int }}|\mathrm{GS}\rangle$ produces $|\mathrm{OpEx}\rangle \propto(|20\rangle-|02\rangle)$, an energy eigenstate [20]. Using an overly simplified basis set, one might falsely presume that the twisting action of the optically excited state comes from the energetics of the antibonding state $|\mathrm{AB}\rangle$. However, the optically excited state has no $|\mathrm{AB}\rangle$ part: Photoexcitation always produces a particle-hole pair [14], not the antibonding state.

The ground-state energy $E_{\mathrm{GS}}^{\pi}(\theta)$ of the $\pi$ electrons is

$$
E_{\mathrm{GS}}^{\pi}(\theta)=\frac{1}{2}\left[(U+V)-\sqrt{(U-V)^{2}+16 t^{2} \cos ^{2} \theta}\right] .
$$

For clarity of presentation, the dynamical bond-stretching variable $u$ is set to zero here, but it is fully included in our calculations. The ground state has energy minima at $\theta=0^{\circ}$ or $\theta=180^{\circ}$ where $\pi$ orbitals are aligned.

In our calculations, we use optimized values $[14,18,21]$ to fix electronic parameters $t=2.5 \mathrm{eV}, \alpha=4.0 \mathrm{eV} / \AA, U=10$ $\mathrm{eV}$. With these parameters, photoexcitation $(7.1 \mathrm{eV})$ and triplet excitation $(4.4 \mathrm{eV})$ energies are in good agreement with experiment. Equation (6) implies a torsional spring constant near $\theta=0^{\circ}$ of

$$
K_{\theta}^{\pi}=\frac{8 t^{2}}{\sqrt{(U-V)^{2}+16 t^{2}}} \approx 4.8 \mathrm{eV} / \mathrm{rad}^{2}
$$

and a torsional barrier of

$$
\begin{aligned}
\Delta E^{\pi} & \equiv E_{\mathrm{GS}}^{\pi}\left(90^{\circ}\right)-E_{\mathrm{GS}}^{\pi}\left(0^{\circ}\right), \\
& =\frac{1}{2}\left[-|(U-V)|+\sqrt{(U-V)^{2}+16 t^{2}}\right] \approx 3.5 \mathrm{eV} .
\end{aligned}
$$

Ground-state measurements tell a different story about the energy surface than does $E^{\pi}$ alone, however. Raman spec-
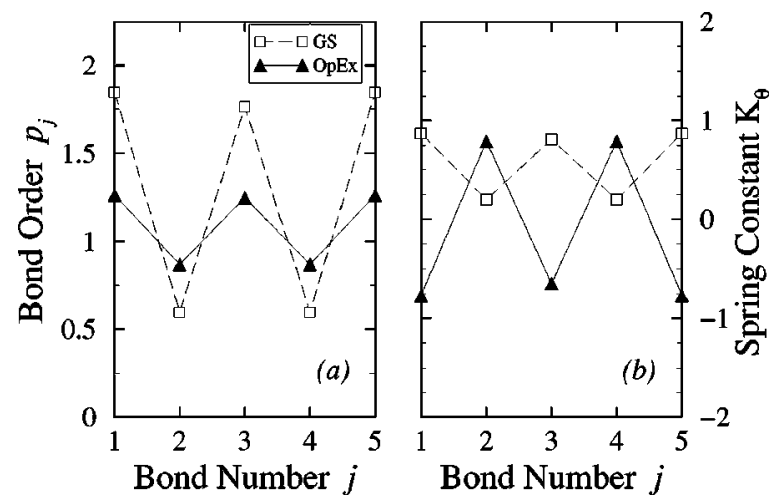

FIG. 3. (a) The bond order $p_{j}$, defined in Eq. (9), is shown for ground and optically excited states in hexatriene $\left(\mathrm{C}_{6} \mathrm{H}_{8}\right)$. (b) The torsional stiffness of the $\pi$ bonds is reduced by torsionally destabilizing steric interactions resulting in the observed ground-state spring constants [25]. Photoexcitation makes the molecule unstable ( $K_{\theta}$ is negative) to twisting about bonds 1,3 , and 5 .

troscopy yields $K_{\theta}=3.54 \mathrm{eV} / \mathrm{rad}^{2}$ and thermal torsional barrier measurements give $\Delta E=2.8 \mathrm{eV}$ [22]. How may these be reconciled with $E^{\pi}$ ?

We attribute the difference to steric interactions [23]. Thus, the net torsional potential observed in Raman spectroscopy is the sum of a stabilizing $\pi$-electronic part and a destabilizing steric part [see Fig. 2(a)]. Individually these effects are both well known; yet combining them and acknowledging their competition explains why effective potentials look so different from the electronic part alone. We use ground-state information $\left(K_{\theta}^{\mathrm{st}}=K_{\theta}-K_{\theta}^{\pi}\right.$ and $\Delta E^{\mathrm{st}}=\Delta E$ $-\Delta E^{\pi}$, with centers of C-H orbitals taken to be $0.7 \AA$ away from the carbon), we obtain steric constants $A=6.7 \AA^{-1}$ and $D=(1 \mathrm{eV}) \exp \{-A \times 2.0 \AA$. The relatively large repulsive radius reflects that, in addition to Pauli exclusion, there is also a significant Coulomb repulsion of the $\sigma$ bonds. We will show next how photoexcitation tips the balance in favor of sterics and leads to a mechanism for isomerization.

In Fig. 2(a), one sees that if not for the destabilizing influence of steric interactions, the optically excited state would not tend to twist: $E_{\mathrm{OpEx}}^{\pi}(\theta)$ is constant. However, with the sterics parametrized from ground-state properties, it takes approximately $30 \mathrm{fs}$ for photoexcited ethylene to twist to $\theta$ $=90^{\circ}$ [see Fig. 2(b)] [24]. This excellent agreement with the measurement [4] and a robustness to parameter changes gives us confidence in the simple scenario that emerges with EHMM, namely that the mechanism of photoisomerization is simply a change in stability, following the deactivation of the torsional stiffness from the $\pi$ electrons.

The EHMM can also be used to study longer polyenes. The torsional stiffness arising from $\pi$ bonding can again be calculated. The effective torsional spring constant is

$$
K_{\theta_{j}}^{\pi}=\left\langle\frac{\partial^{2} H_{\pi}}{\partial \theta_{j}^{2}}\right\rangle_{\theta_{j}=0} \approx\left(t-\alpha u_{j}\right) p_{j},
$$

where $p_{j} \equiv \Sigma_{s}\left\langle c_{j+1, s}^{\dagger} c_{j, s}+\right.$ H.c. $\rangle$. For example, in the ground state of cis-hexatriene $\left(\mathrm{C}_{6} \mathrm{H}_{8}\right)$, the $\pi$ electrons again provide 
much greater torsional stiffness than observed from vibrational spectra [25] [see Fig. 3(b)].

The steric spring constants can be estimated as before: $K_{\theta}^{\mathrm{st}}=K_{\theta}-K_{\theta}^{\pi, \mathrm{GS}}$, with $K_{\theta}^{\pi}$ as calculated in Eq. (9) and $K_{\theta}$ as measured spectroscopically [25]. The steric values are consonant with those found in ethylene, increased somewhat by the increased number of destabilizing interactions. As Fig. 3 illustrates, photoexcitation reduces the bond order for double bonds and increases it for single bonds. In this way photoexcitation alters the torsional stiffness profile - leading to a net destabilization of the central cis double bond, at least intially. Preliminary studies along these lines with more complicated molecules, including rhodopsin [15], are promising.

To summarize, we have investigated the dynamics of conjugated polyenes in both the ground and the optically excited states using the realistic ingredients of the EHMM. Solving the fully correlated quantum mechanics exactly, with care taken to find the true optically excited state, is a straightforward but significant advancement; thus, we can now com- pute the coherent photoexcited state. EHMM reveals surprisingly deep insights about the old question of photoisomerization.

A clear understanding of the competition between model elements $-\pi$ electronics and sterics - is necessary to resolve discrepancies in the ground state between effective force fields found by analyzing vibrational spectra and those inferred from electronic couplings alone. The scenario that emerges is that steric forces between nonbonded $\sigma$ orbitals play a large destabilizing role and that the ultrafast coherent dynamics that leads to photoisomerization in these cases results simply from a change in stability. This mechanism is enhanced by spatial symmetry breaking in rhodopsin and other ultrafast systems.

We thank Huub de Groot for introducing us to the problem and for stimulating discussions. We thank Henk Eskes for shedding some light on optical excitations and Peter Denteneer and Jonathan Pyle for discussions. We also thank Martin Karplus for bringing early work to our attention. B.F.G. acknowledges support from NSF-REU.
[1] R. Hubbard and A. Kropf, Proc. Natl. Acad. Sci. USA 44, 130 (1958).

[2] R. W. Schoenlein et al., Science 254, 412 (1991).

[3] R. W. Schoenlein et al., J. Phys. Chem. 97, 12087 (1993).

[4] P. Farmanara, V. Stert, and W. Radloff, Chem. Phys. Lett. 288, 518 (1998).

[5] Q. Wang et al., Science 266, 422 (1994).

[6] F. Bernardi, M. Olivucci, and M. A. Robb, Chem. Soc. Rev. 25, 321 (1996).

[7] E. V. Tsiper et al., J. Chem. Phys. 110, 8328 (1999).

[8] H. J. M. de Groot (private communication).

[9] W. Bialek, R. F. Goldstein, and S. Kivelson, in Structure, Dynamics, and Function of Biomolecules, edited by A. Ehrenberg et al. (Springer, Heidelberg, 1987).

[10] A. Bifone, H. J. M. de Groot, and F. Buda, Chem. Phys. Lett. 248, 165 (1996); Pure Appl. Chem. 69, 2105 (1997).

[11] J. Michl and V. Bonacic-Koutecky, Electronic Aspects of Organic Photochemistry (Wiley, New York, 1990).

[12] L. Salem and C. Rowland, Angew. Chem. Int. Ed. Engl. 11, 92 (1972).

[13] A. Warshel and M. Karplus, J. Am. Chem. Soc. 94, 5612 (1972); Chem. Phys. Lett. 32, 11 (1975); A. Hemley et al., J. Phys. Chem. 92, 945 (1988).

[14] A. J. Heeger et al., Rev. Mod. Phys. 60, 781 (1988); Yu Lu, Solitons \& Polarons in Conducting Polymers (World Scientific, Singapore, 1988).

[15] D. P. Aalberts, F. L. J. Vos, and W. van Saarloos, Pure Appl. Chem. 69, 2099 (1997).
[16] U. Burkert and N. L. Allinger, Molecular Mechanics, ACS Monograph No. 177 (American Chemical Society, Washington, DC, 1982).

[17] S. R. White, Phys. Rev. B 48, 10345 (1993); M. Boman and R. J. Bursill, ibid. 57, 15167 (1998).

[18] W. Barford, R. J. Bursill, and M. Yu. Lavrentiev, J. Phys.: Condens. Matter 10, 6429 (1998).

[19] G. D. Mahan, Many-Particle Physics (Plenum, New York, 1990).

[20] Generally $|\mathrm{OpEx}\rangle$ is not an energy eigenstate in longer polyenes: D. P. Aalberts et al. (unpublished).

[21] W. A. Harrison, Electronic Structure and the Properties of Solids (Freeman, San Francisco, 1980).

[22] L. Giroux, M. H. Back, and R. A. Back, Chem. Phys. Lett. 154, 610 (1989).

[23] Steric forces in polyenes are often neglected except when imposed by a cis bond [e.g., A. Warshel and M. Karplus, J. Am. Chem. Soc. 96, 5677 (1974); or Ref. [15]]. Even when torsional destabilization around a bond has been considered, it was as a "purely formal device", [e.g., U. Dinur, A. Hemley, and M. Karplus, J. Phys. Chem. 87, 924 (1983)]. We emphasize both the importance and the physical nature of these steric interactions.

[24] Ab initio calculations indicate a 50-fs photoisomerization time: M. Ben-Nun and T. J. Martinez, Chem. Phys. Lett. 298, 57 (1998).

[25] F. Negri et al., J. Chem. Phys. 90, 5944 (1989). 